\title{
Structural Assessment of (Sub-)Monolayer Coatings in Device Processing at High Spatial Resolving Power by TOF-SIMS Tandem MS Imaging
}

\author{
Gregory L. Fisher ${ }^{1}$ \\ 1. Physical Electronics, Chanhassen, Minnesota, USA.
}

Whether in discovery research, failure analysis or reverse engineering, the objective is to ascertain the 2D/3D composition and structure of molecules in devices as a result of various processing steps. Most often there is neither sufficient background information nor reference spectra for substantiation of the analytical observations. Two case studies are described here wherein little guidance was provided but accurate and concrete answers were desired. In one case, a carbon residue was observed by Auger electron spectroscopic (AES) imaging of etched $\mathrm{e}^{-}$beam-patterned structures, but the source of carbon was indeterminate. In a second case, molecules having ligands were patterned by a photolithography method and ostensibly loaded with catalytically active metals. Spatially resolved characterization was performed, but fell short of verifying the presence and structure of the intact metal-organic structures.

In this report, a general time-of-flight secondary ion mass spectrometry (TOF-SIMS) imaging method for unknown identification and structure elucidation is demonstrated, including the aforementioned examples. This method makes use of a TOF-TOF spectrometer designed for simultaneous TOF-SIMS (MS ${ }^{1}$ ) imaging and tandem MS (MS ${ }^{2}$ imaging [1-4]. Ions for TOF-SIMS tandem MS imaging were produced by a $\mathrm{Bi}_{3}{ }^{+}$ primary ion nanoprobe whereby $\mathrm{MS}^{1}$ and $\mathrm{MS}^{2}$ spectral data were generated from the same pixel in one duty cycle at a resolving power of $300 \mathrm{~nm}$. Ion fluences were within the static limit (i.e. $<10^{13} \mathrm{Bi}_{3}{ }^{+} / \mathrm{cm}^{2}$ ) for nondestructive analysis, even where multiple analyses were conducted at the same position of the sample. Tandem MS peak attributions were made to a calculated mass accuracy $\left(\Delta_{m / z}\right)$ of $\approx 1 \mathrm{ppm}$ which provides confidence in the accuracy of the molecular identifications.

In the first example, TOF-SIMS tandem MS imaging was applied to characterize the carbon-containing residues of the lithography-patterned and etched structures (Figure 1). Several sets of peaks were observed at $m / z>150$ in the $\mathrm{MS}^{1}$ spectra. It was established and verified by tandem MS imaging that the carbon observed by AES imaging on the patterned sidewalls arose from two fatty acid (FA) contaminants; specifically, the $[\mathrm{M}-\mathrm{H}]^{-}$ions of FA(12:0) and FA(16:0) were identified. Further, a $\approx 2: 1$ ratio of FA(12:0) to FA(16:0) was calculated. The high $\mathrm{m} / z$ peaks, detected only on the $\mathrm{Cu}$-plated surfaces, were attributed to metal-organic complexes of $\mathrm{Cu}$ with the fatty acids. In the positive ion polarity, $\mathrm{FACu}_{2}{ }^{+}$and $\mathrm{FA}_{2} \mathrm{Cu}_{3}{ }^{+}$ ions were identified; in the negative ion polarity, $\mathrm{FA}^{-}$and $\mathrm{FA}_{2} \mathrm{Cu}^{-}$ions were identified. In each case, a natural $\mathrm{Cu}$ isotope composition was confirmed.

TOF-SIMS tandem MS imaging was also employed to confirm the presence and elucidate the structure of metal-organic ligands of $\mathrm{Au}, \mathrm{Pd}$ or Pt atoms attached to bipyridine or triphenylphosphine within patterned monolayer films (Figure 2). Metal atom loading was achieved after monolayer immobilization [5]. The pattern areas were readily observed based on the atomic metal ion signatures. There were a number of $\mathrm{MS}^{1}$ precursor ions potentially related to the metal-organic ligands based on the $m / z$ values lying in the anticipated range for the target structures. The 2D distribution and structure of complexed bipyridine and triphenylphosphine ligands, together with observing the natural isotope distribution of the metals, was achieved by tandem MS imaging [6]. 
References:

[1] GL Fisher et al., Anal. Chem. 88 (2016), p. 6433.

[2] GL Fisher et al., Microscop. Microanal. 23 (2017), p. 843.

[3] CE Chini et al, Biointerphases 13 (2018), p. 03B409.

[4] T Fu et al., Nature Sci. Rep. (2018) accepted December 06, 2018.

[5] R Müller et al., Chemistry (2018). DOI: 10.1002/chem.201803966.

[6] R Müller and A Welle at Karlsruhe Institute of Technology (KIT) and C Barner-Kowollik at Queensland University of Technology (QUB) are acknowledged for providing the samples for analysis.
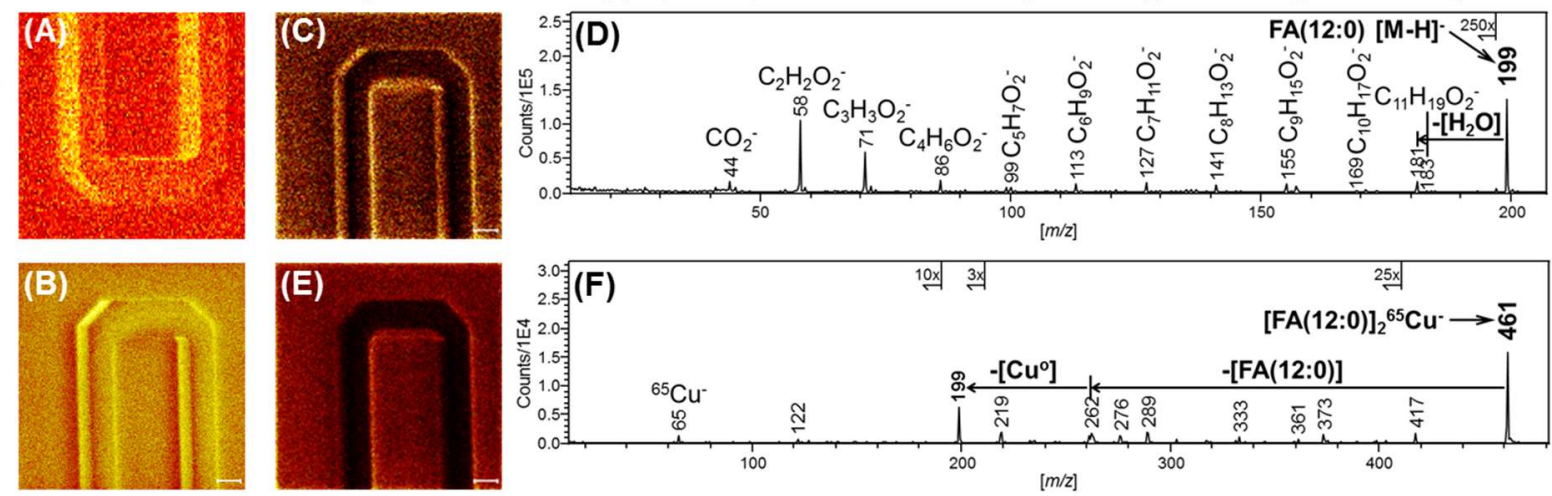

Figure 1. (A) An AES map of carbon on an $\mathrm{e}^{-}$beam patterned device structure after etching. The signal of carbon is elevated on the sidewalls of the structure where there is no $\mathrm{Cu}$ plating. The image area is 70 $\mu \mathrm{m} \times 70 \mu \mathrm{m}$, and the image is rotated $180^{\circ}$ to display the same sidewall illumination as viewed in the TOF-SIMS images. (B) An $\mathrm{MS}^{1}$ ion image of $\mathrm{SiCO}_{2} \mathrm{H}^{-}\left(\mathrm{m} / z\right.$ 73) in a $100 \mu \mathrm{m} \times 100 \mu \mathrm{m}$ area of the $\mathrm{e}^{-}$beam patterned device structure after etching. This image and others, e.g. $\mathrm{C}_{2}^{-}(\mathrm{m} / z 24)$ and $\mathrm{SiCH}^{-}(\mathrm{m} / z$ 41), show elevated carbon moieties at the sidewalls of the structure. (C) An $\mathrm{MS}^{2}$ ion image of $\mathrm{m} / z 199$ ions, identified as $\mathrm{FA}(12: 0)[\mathrm{M}-\mathrm{H}]^{-}$by the product ion spectrum in panel (D), indicating a greater abundance at the sidewalls of the structure. (E) An $\mathrm{MS}^{2}$ ion image of $m / z 461$ ions, identified as $[\mathrm{FA}(12: 0)]_{2}{ }^{65} \mathrm{Cu}^{-}$by the product ion spectrum in panel $(\mathrm{F})$, revealing that the metal-organic complex is present only at the $\mathrm{Cu}-$ plated surface of the device structure.
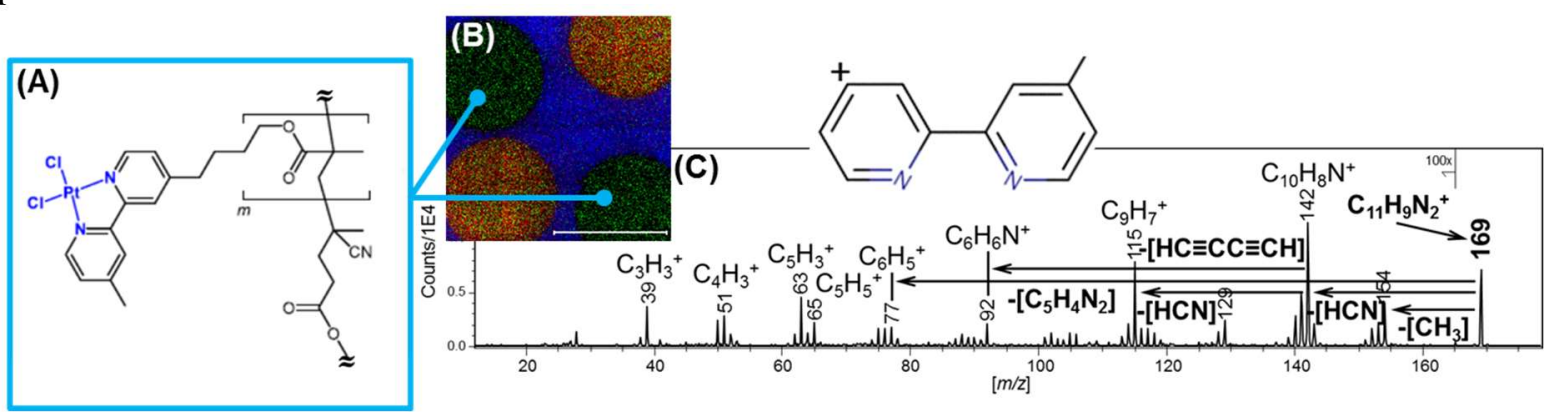

Figure 2. (A) Illustration of a metal-organic ligand of the bi-functionalized monolayer and where it is observed within the imaged area. (B) False color overlay ion image of the bi-functionalized monolayer composed of $\mathrm{Pd}^{+}\left(\mathrm{MS}^{1} m / z\right.$ 106, red), $\mathrm{C}_{11} \mathrm{H}_{9} \mathrm{~N}_{2}{ }^{+}\left(\mathrm{MS}^{2} \mathrm{~m} / z\right.$ 169, green) and $\mathrm{SiH}^{+}\left(\mathrm{MS}^{1} \mathrm{~m} / z\right.$ 29, blue). The scale marker is $1 \mathrm{~mm}$. (C) An $\mathrm{MS}^{2}$ product ion spectrum of the presumed 4-methyl-2,2'-bipyridine moiety of the metal-organic ligand depicted in panel (A) which was acquired simultaneously with the image data shown in panel (B). The product ion spectrum of the $m / z 169$ precursor ion (inset) is consistent with the 4-methyl-2,2'-bipyridine composition and the peak attributions are made to a calculated mass accuracy $\left(\Delta_{m / z}\right)$ of $+1.31 \mathrm{ppm}$. 